\title{
Temperature and Magnetic Resonance Characteristics of Zinc, Manganese, Gadolinium, Gold, Iron Magnetic Nanoparticles and Cytokine Synergy in Hyperthermia
}

\author{
Saleh S. Hayek ${ }^{1}$, Rakesh Sharma ${ }^{1}$, Soonjo Kwon ${ }^{2}$, Avdhesh Sharma ${ }^{3}$, Ching J.Chen ${ }^{1}$ \\ ${ }^{1}$ College of Engineering, King Faisal University, Al-Hasa, P.O. Box 380, Zip 31982. ${ }^{2}$ Department of Biological Engineering, Utah State University, Logan, UT \\ 84322. ${ }^{3}$ Nanotechnology Division, Electrical Engineering Department, MP University of Agriculture and Technology, Udaipur, Rajasthan, India. Correspon- \\ dence should be addressed to Rakesh Sharma (rksz2004@gmail.com) \\ Received June 30, 2008; revised October 18, 2008; accepted October 18, 2008
}

\begin{abstract}
The temperature sensitive magnetic resonance dependence for assessing localized heating effect of Manganese (Mn), Zinc (Zn), Gadolinium(Gd), Gold(Au) and Iron(Fe) magnetic nanoparticles was compared. These particles showed heating effect when subjected to alternating filed. The relationship between temperature and magnetic nanoparticle moment is specific in imaging. The art of imaging temperature in a tumor at various locations is emerging as the selective approach of hyperthermia to monitor temperature and treat the tumor. Two unsolved issues are related with tumor temperature rise in the presence of magnetized nanoparticles. First, the relationship of tumor energy changes as a result of cytokine synergy after tumor magnetization. The second issue is linear attenuation after magnetic field exposure with tissue temperature increase due to inflammation and lysosomal enzyme action in tumor. In present paper, a new approach of heating tumor is analyzed without spot heating by polymer coated particles at controlled Curie temperature of less than $44^{\circ} \mathrm{C}$. The study reports a comparison of $\mathrm{Mn}, \mathrm{Zn}, \mathrm{Gd}, \mathrm{Fe}, \mathrm{Au}$ nanoparticles designed for imaging purpose using chemical co-precipitation technique. The possibility of nanoparticle stimulated temperature treatment (hypothermia effect) is hypothesized to recover the tumor metabolic integrity and inflammatory status of tumor cells presumably associated with the depleted intracellular energy (low ATP) and the inflammation (elevated cytokines, interleukins) and lysosomal enzymes initially. The multimodal imaging techniques were compared using nanoparticles for their sensitivity. The art of the nanoparticle induced hyperthermia does have a great impact
\end{abstract}

on public health as alternative therapeutic oncology.

Keywords: hyperthermia, nanoparticles, temperature imaging, cytokine

\section{INTRODUCTION}

Heating tumors by nanoparticles and resistance in hypoxic tumor cells to a high temperature is emerging as an effective tool in therapeutic oncology [1]. Heating of organs and tissues in cancer treatment was first reported [2]. The introduction of nanoparticles enhanced the diagnosis and localization of specific tumor characteristics by multimodal imaging techniques including optical, magnetic resonance, positron emission tomography, computed tomography and X-ray techniques. With rapid development, feasible clinical therapeutic applicators and hyperthermia equipment were designed. In this direction, colloidal gold-thiol preparations were first reported as effective staining agents to label proteins in both diagnostics such as imaging, blotting, flow cytometry, hybridization assays and gold-thiol hyperthermia agents. Other potential hyperthermic particles are silver, iron, zinc and lanthanum nanoparticles [3-10].

At sites of tumor, hyperthermia is a state of therapeutic temperature induced recovery of metabolic integrity and enough defense by cytokines and lysosomes to compensate the damage perhaps increase in oxygenated blood circulation across tumors due to stimulus of heat. In normal tumor, the cells experience loss of metabolic integrity, hypoxia, inflammation, stimulated lysosomal enzymes due to phagocytosis. As a result tumor cells get overactive and overgrown. The hyperthermia can be produced by the interaction of tumor cells with energy such as alpha-, beta- or gamma radiation (Therapeutic Nuclear Medicine), X-Ray (radiotherapy), ultrasound (Therapeutic Ultrasonography) and magnetization (magnetotherapy). However, these interactions are not risk free. Alter- 


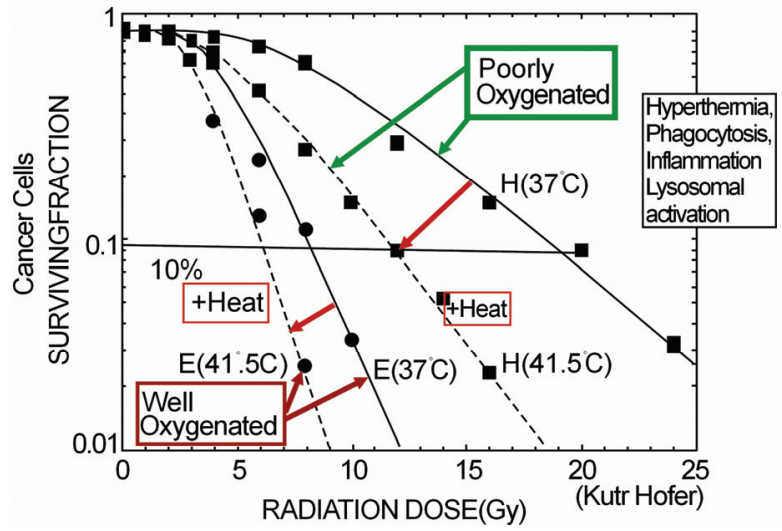

Figure 1. The effective radiation therapy is shown to change the oxygenated tumor tissue. (Note the oxygen deficient hypoxic cells (on right side of the graphs) are resistant to radiation than normal cells.)

native common practice of radiation therapy puts hazard of oxygen radical formation and tumor's cell DNA strand breakage. However, oxygen-starved hypoxictumor cells are three-times more resistant to ionizing ra diation than are normal cells [8]. As a result, hypoxic cells show failure in achieving local tumor control through ionizing radiation hyperthermia treatments as shown in Figure 1 and reported elsewhere [9-10]. Good catch is here to enhance the magnitude of heating effect in tumor by either of the energy type using nanoparticles. This alternative approach can be useful in imagingsuch as "magnetic hyperthermia for MRI" or "gold hyperthermia for X-Ray" or "Acoustic hyperthermia for ultrasound" by introducing these particles uniformly distributed in tumors.

In recent years, these particles have been synthesized to magnify the tissue heating effect. However, hyperthermia may be hazard of spot overheating that causes necrosis. Magnetic hyperthermia is the method of heating body tissue using magnetic materials [11, 15, 16]. The overheating may be avoided by controlling the particles' dissipating heating effect at their Curie temperature. Presently, colloidal gold particles are used in monitoring metabolic changes as are bound with antibodies, lipids, DNA fragments during cellular uptake and endocytosis. In human body, nanomagnetic particles with Curie temperature of $42-43^{\circ} \mathrm{C}$ are most suitable as hyperthermia heat source at the tumor site exposed to an alternating field [11].

In our lab, a simple resonant circuit was developed for nanoparticles heating effect after applied alternating fields to generate hyperthermia as shown in Figure 2. The particle behave is specific to Curie temperature. At Curie temperature, particles generate consistent heat at the applied alternating fields to maintain tumor heating [14-15]. Mn-Zn-Gd Ferrite nanoparticles have advantage as their properties can be desirable by varying the proportions of the constituent $\mathrm{Mn}$ or $\mathrm{Zn}$ or Gd elements [1719].

In this feasibility paper, our focus is on the use of different techniques that were used in our lab. Such tech-

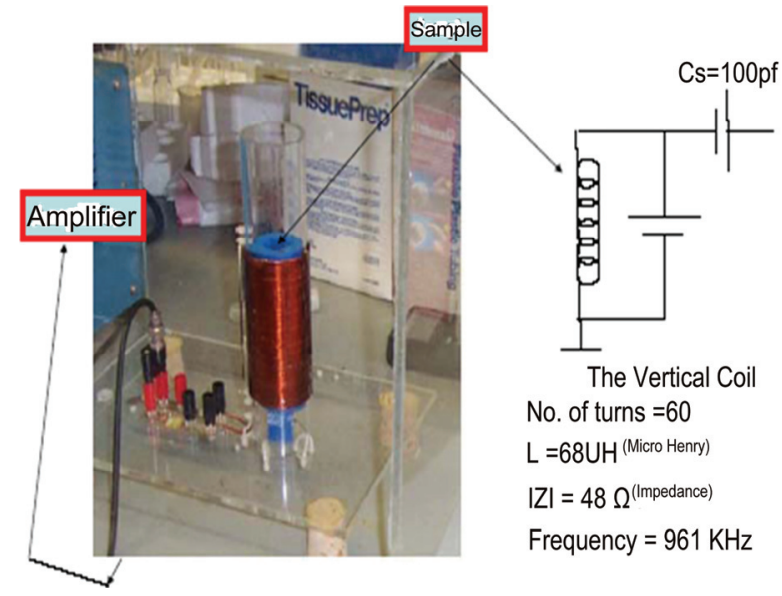

Figure 2. The photograph of resonant circuit is shown with sample holder and amplifier (on left) and design of vertical coil with its specifications (on right).

niques are: 1 . synthesis, targeting the tumor and the relaxation characteristics of nanoparticles in the tissue; 2. Mechanistic approach of cytokines and lysosomal enzymes in tumors and imaging the nanoparticle concentrations in the tumors; 3. Comparison of newer nanoparticles in hyperthermia and emerging biomedical applications. This study further demonstrates three main interactions contributing the measurable signal: 1 . Tumor tissue relaxation behavior and temperature dependence; 2 . Nanoparticles relaxation behavior and temperature dependence; 3. Intracellular tumor cell biochemical- NMR relationship. The paper also shows a comparison of manganese, zinc, gadolinium, ferrite, gold and lanthanum nanomagnetic particles and their magnetic moment dependence on temperature to highlight the significance of Curie temperature of $42-43^{\circ} \mathrm{C}$ in hyperthermia. The emerging newer concepts are revisited if cytokine synergy and energy metabolic integrity of tumor cells participate in temperature rise in presence of nanoparticles in tumor. The novelty of this method is the magnetic moments achieved at varying proportions of particles at the Curie temperature of the nanoparticles are specific to generate heating effect(hyperthermia) and visible magnetization or T1 relaxation constants (for MRI) or Hounsfield Number(for X-Ray).

Newer Concepts of Tumor Inflammation and Hyperthermia:

In initial stage of tumor, cells experience the events of hypoxia (low oxygen and low oxidative phosphorylation); progressive inflammation (cytokine synergy); heat shock protein element(gene) trigger to synthesize cAMP mediated heat shock proteins with lysosomal stimulation (cathepsins in pro-apoptosis). Advanced stage of tumor shows high glycolysis; high oxygen/ATP demand; elevated cytokines, interleukines, however, subsequent recovery by lysosomal enzymes (towards normal) leads to less tumor cell death and high tumor vascularization (low inflammation, less energy demand and normal temperature). We postulate that hyperthermia may cause supply of enthalpy heat (activation energy) to lysosomal en- 
zymes at normal rate; keeping normal cytokines and humoral immunity as most important event.

The heating power area under loop enclosed by hysteresis was calculated by heating power dissipated by particles in magnetic field, $\mathrm{H}$ as following:

$$
\mathrm{P}=\mathrm{f} . \mathrm{M} \cdot \mathrm{dH}
$$

where $\mathrm{f}$ is frequency of $\mathrm{AC}$ magnetic field and M.dH is hysteresis loop area as shown in Figure 3.

\section{MATERIALS AND METHODS}

The batch method of co-precipitation was adopted as previously described by Saleh et al.[12] The particles in the form of complexes were synthesized by chemical coprecipitation method. We present here a model $\mathrm{Zn}_{\mathrm{x}} \mathrm{Gd}_{\mathrm{x}} \mathrm{Mn}_{(1-\mathrm{x})} \mathrm{Fe}_{3} \mathrm{O}_{4}$ chemical co-precipitation method using different $\mathrm{Gd}$ proportions (x) such as $\mathrm{Mn}_{0.5} \mathrm{Zn}_{0.5} \mathrm{Gd}_{\mathrm{x}} \mathrm{Fe}_{(2-\mathrm{x})} \mathrm{O}_{4}$. In our lab, the following method was standardized as:

(1) $0.1 \mathrm{M}$ solution of the metal salts $\mathrm{FeCl}_{3}, \mathrm{Fe}_{2} \mathrm{SO}_{4}$, $\mathrm{ZnSO}_{4}$ and $\mathrm{GdCl}_{3}$

(2) Added to an $8 \mathrm{M}$ solution of $\mathrm{NaOH}$.

(3) The mixture was stirred vigorously at $90^{\circ} \mathrm{C}$ for 40 minutes.

(4) The synthesized $\mathrm{Zn} \mathrm{Gd} \mathrm{Fe} \mathrm{nanoparticles} \mathrm{filtered} \mathrm{with}$ Size up to $10 \mathrm{~nm}$.

(5) Washed 3 times with distilled water and 3 times with acetone.

(6) The particles allowed to dry in nitrogen gas at room temperature.

\subsection{Polymer PEG Encapsulation for Nano- spheres}

A following batch process was developed using above steps for preparing composite particles as described initially elsewhere [12]. Poly ethylene glycol PEG was used to encapsulate Mn.Zn.Fe and Zn.Gd.Fe nanoparticles to improve their biocompatibility. Encapsulation of Mn.Zn.Fe and Zn.Gd.Fe nanoparticles was performed using polymer emulsion method patented by modified solvent evaporation method. The ingredients were used: Polyethylene glycol (PEG) MW: 1,540: 2 gm (polymer), Methylene Chloride: $10 \mathrm{ml}, 13.2$ gm (solvent), Water: 40 $\mathrm{ml}$ (Aqueous medium), Sodium dodecyl sulphate: 0.33 gm (Emulsifying agent), 1-Octanol: $1.1 \mathrm{ml}, 1.32 \mathrm{gm}$ (Inhibitor compound) and Mn.Zn.Fe and Zn.Gd.Fe particles: $50 \mathrm{mg}$. The magnetic particle: polymer ratio was approximately 1:40. The sodium dodecyl sulphate and 1octanol were dissolved in $40 \mathrm{ml}$ of distilled water using a magnetic stirrer. Later $50 \mathrm{mg}$ of Mn.Zn.Fe / Zn.Gd.Fe was added. The polymer phase was prepared by dissolving $2 \mathrm{gm}$ of PEG into $10 \mathrm{ml}$ of methylene chloride. A crude emulsion was formed by adding the polymer phase to the aqueous medium phase. It was sonicated using an ultrasonicator 5 times in steps of 3 minutes and stirred inside a round bottom flask for 12 hours at $700 \mathrm{rpm}$. The solvent was then removed using vacuum evaporation method. The polymer encapsulated particles formed were washed with acetone and stored under PBS buffer solution.

These Mn-Zn-ferrite particles and Gd substituted MnZn-Ferrite particles were obtained via chemical coprecipitation and ferritization. First the metal salts were co-precipitated into hydroxides. This was done by addition of aqueous solution of metal salts in water to the co precipitating base (e.g. $\mathrm{NaOH}, \mathrm{CH}_{3} \mathrm{NH}_{3} \mathrm{OH}$ etc.). For the case of $\mathrm{Mn}-\mathrm{Zn}$ Ferrite particles the reaction occurs as follows:

$(1-\mathrm{x}) \mathrm{Mn}^{2+}+\mathrm{xZn}^{2+}+2 \mathrm{Fe}^{3+}+8 \mathrm{OH}^{-} \longrightarrow(1-\mathrm{x}) \mathrm{Mn}(\mathrm{OH})_{2}$. $\mathrm{xZn}(\mathrm{OH})_{2} \cdot 2 \mathrm{Fe}(\mathrm{OH})_{3}$

Soon after, this precipitate was transformed into ferrite by heating it in the precipitation alkaline solution (ferritization). The reaction for $\mathrm{Mn}-\mathrm{Zn}$ ferrite particles was as follows:

$(1-\mathrm{x}) \mathrm{Mn}(\mathrm{OH})_{2} \cdot \mathrm{xZn}(\mathrm{OH})_{2} \cdot \mathrm{Fe}(\mathrm{OH})_{3} \longrightarrow \mathrm{Mn}_{(1-}$ x) $\mathrm{Zn}_{\mathrm{x}} \mathrm{Fe}_{2} \mathrm{O}_{4} \cdot \mathrm{nH}_{2} \mathrm{O}+(4-\mathrm{n}) \mathrm{H}_{2} \mathrm{O}$

\subsection{Measurement of Reaxation Constants in Nanometals and Tumor Tissues}

The measurement of inverse longitudinal (1/T1) and inverse transverse relaxation (1/T2) constants of nanometals in soplutions and tumor excised tissues was performed on Bruker-Spec 60 desktop model as described elsewhere [15]. Different chemicals $\mathrm{FeCl}_{3} \cdot 6 \mathrm{H}_{2} \mathrm{O}, \quad \mathrm{GdCl}_{3} \cdot 6 \mathrm{H}_{2} \mathrm{O}, \quad \mathrm{MnCl}_{2} \cdot 4 \mathrm{H}_{2} \mathrm{O}$ and $\mathrm{ZnSO}_{4} .7 \mathrm{H}_{2} \mathrm{O}$ were used to obtain $\mathrm{Fe}^{3+}, \mathrm{Gd}^{3+}, \mathrm{Mn}^{2+}$ and $\mathrm{Zn}^{2+}$ ions in the aqueous solution. This salt solution at $90^{\circ} \mathrm{C}$ was added to $8 \mathrm{M} \mathrm{NaOH}$ solution at $90^{\circ} \mathrm{C}$ followed by vigorous stirring. The stirring and heating at $90^{\circ} \mathrm{C}$ was continued for a minimum of 40 mins. It has been previously reported elsewhere that heating for over 40 mins does not produce any significant changes in the particles properties [17-19]. The product was then filtered, washed with distilled water and finally washed and dried with acetone.

The hysteresis curves were obtained at room temperature using a vibration sample magnetometer (VSM). A Quantum Design SQUID was used to study the temperature dependence of the magnetization [19].

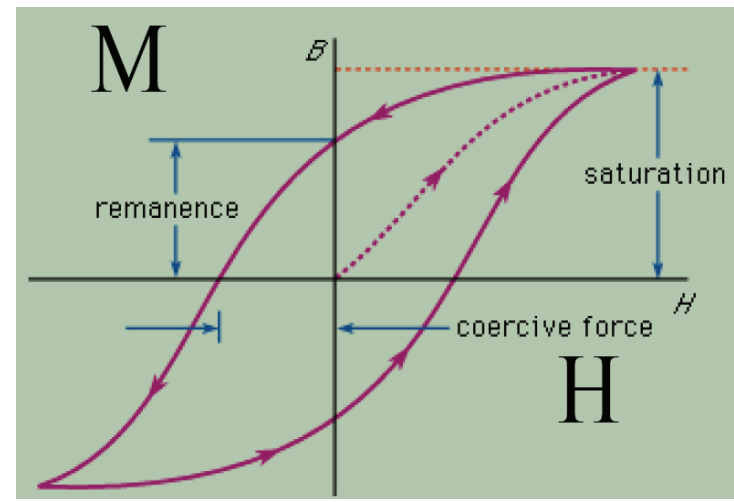

Figure 3. A representative hysteresis loop is shown with different regions of the loop. 

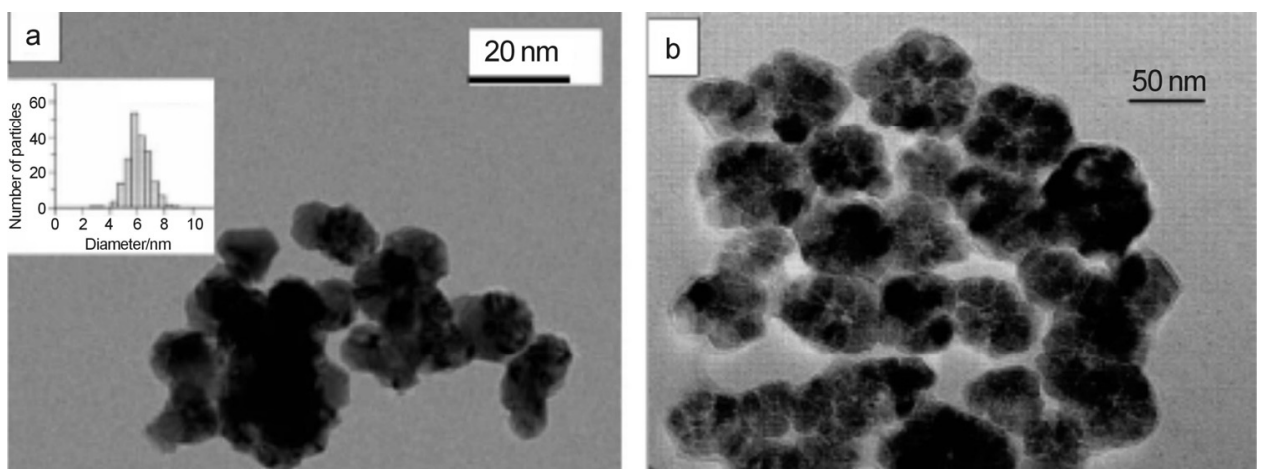

Figure 4. (panel on left) A TEM view of $20 \mathrm{~nm}$ sized Gd-Zn-ferrite nanoparticles is shown with insert of distribution of different sizes of nanopartiles in the high power field (magnification $\times 105$ ). (panel on right) For comparison with earlier report on TEM view of $50 \mathrm{~nm}$ sized CMD-coated iron oxide particles prpared by wet chemical preparation as described elsewhere [27].
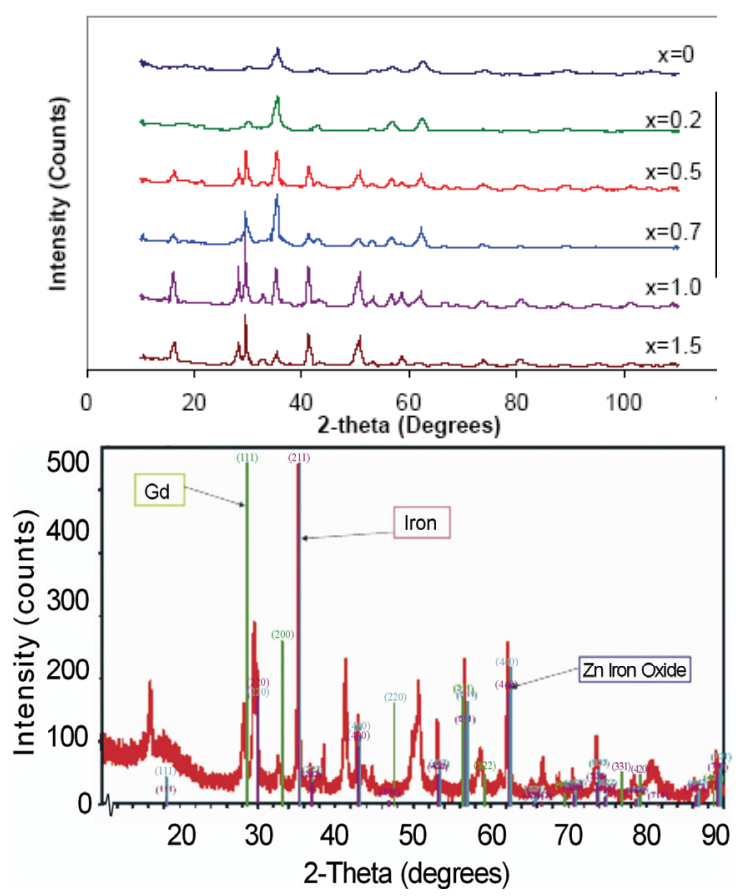

Figure 5. Different samples show specific $X$-ray diffraction peak patterns at different proportions $(x=0,0.2,0.5,0.7,1.0$, 1.5). (At bottom) A representative $X$-ray diffraction peak patterns of standard samples of $\mathrm{Gd}$, iron, $\mathrm{Zn} . \mathrm{Fe} 3 \mathrm{O} 4$ are shown as distinct peaks (ASME IMEC Seattle meeting 2008; Presenter: Dr CJ Chen IMECE 2007-43617). Modified from reference [27]. See for details webpage :http://etd.lib.fsu.edu/theses 1/submitted/etd07082007-135942/unrestricted/Al-HayekSDissertation.pdf

\section{RESULTS}

All the samples were examined by Transmission Electron Microscopy (TEM), X-ray powder diffraction (XRD). The Gd Zn-ferrite nanoparticles and Gd Znferrite HSA $20 \mathrm{~nm}$ sized encapsulated nanoparticles in ethanol were deposited over a $\mathrm{Cu}$ grid as shown by TEM in Figure 4.

Gd Zn-ferrite nanoparticles and Gd-XRD diagrams for the samples are shown in Figure 4. All major peaks were indexed to the standard pattern for Mn-Zn Ferrite.

\subsection{Hysteresis Curves}

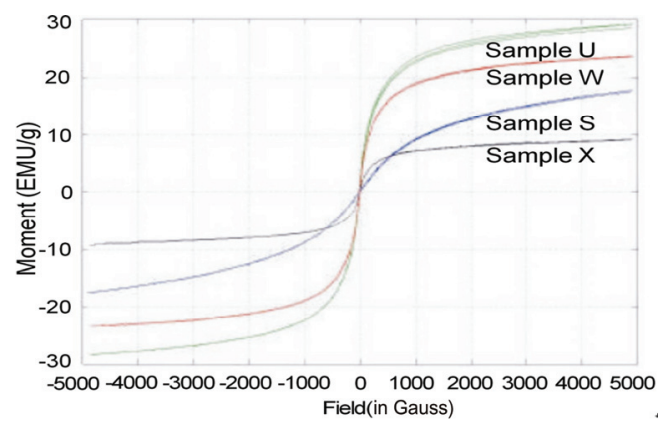

Figure 6. Hysteresis curves of different samples show sigmoid curves at different Gd proportions in the samples (ASME IMEC Seattle meeting 2008: Presenter: Dr CJ Chen IMECE200743617).

Table 1. The table shows the variation in saturation magnetization and Curie temperature with increasing Gd proportion.

\begin{tabular}{|c|cc|}
\hline $\begin{array}{c}\text { Gadolinium } \\
\text { proportion (x) }\end{array}$ & $\begin{array}{c}\text { Curie Temperature } \\
(\mathrm{K})\end{array}$ & $\begin{array}{c}\text { Saturation magnetization } \\
\text { (EMU/g) }\end{array}$ \\
\hline 0 & 320 & 20 \\
0.2 & 409 & - \\
0.5 & 412 & 29 \\
0.7 & 406 & - \\
1.0 & 414 & 24 \\
1.5 & 382 & 9.5 \\
\hline
\end{tabular}

The hysteresis curves at room temperature for the samples with $\mathrm{Gd}(\mathrm{x}=0,0.2,0.5,0.7,1.0,1.5)$ were obtained using a vibration sample magnetometer shown in Figure 5 , by subjecting them to a field in the range of 0 to 5,000 $\mathrm{G}$ as shown in Figure 6. All these samples were ob served as soft-magnetic with specific hysteresis loops as shown in Figure 7 where hysteresis curve is proportional to the energy dissipated in the form of power heating loss.

\subsection{Saturation Magnetization}

The saturation magnetization behavior was specific to gadolinium proportion and temperature dependent. It showed maximum value at gadolinium proportion 0.5 and Curie temperature $412 \mathrm{~K}$ as shown in Table 1. 


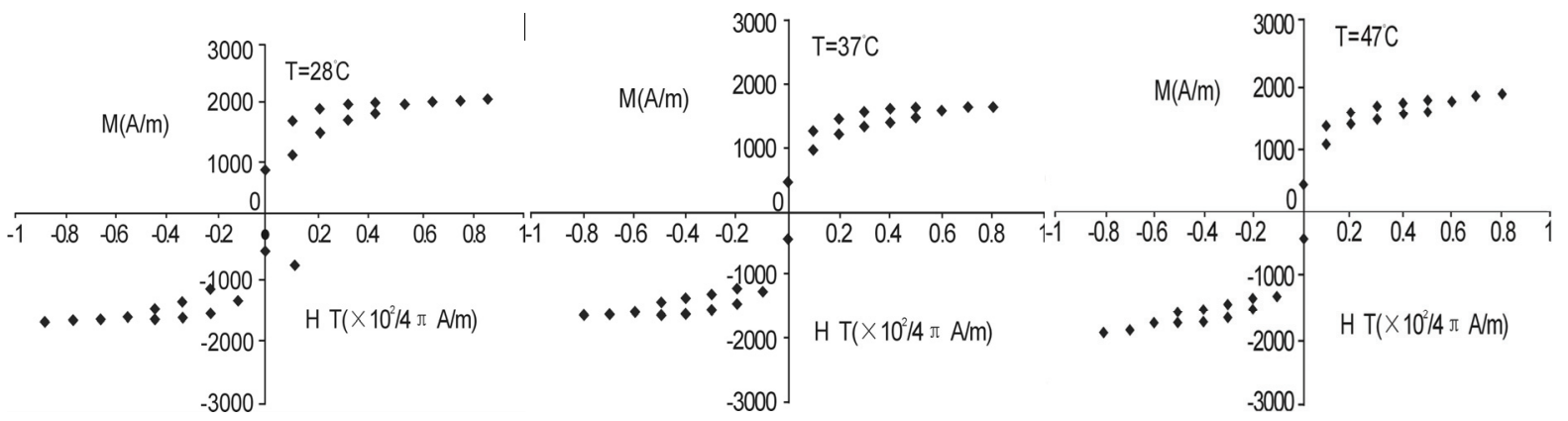

Figure 7. The hysteresis curves are shown at different temperatures of $28^{\circ} \mathrm{C}, 37^{\circ} \mathrm{C}, 47^{\circ} \mathrm{C}$. It can be seen from the figure that the remnant magnetization $\sigma$, the saturation magnetization $\mathrm{Ms}$ and the area of hysteresis loop were found to be decreasing with increasing temperature, e.g., the power loss decreases with increasing temperature. (ASME IMEC Seattle meeting 2008: Presenter: Dr CJ Chen IMECE2007-43617).

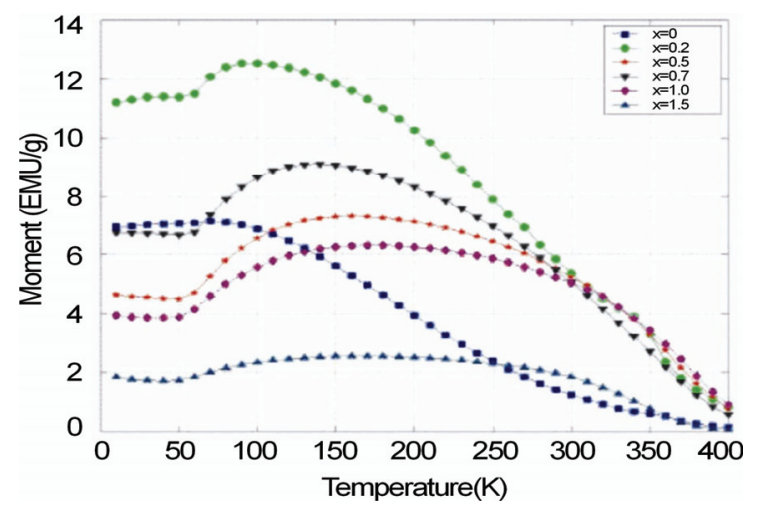

Figure 8. The temperature and magnetic moment relationship is shown for samples with different Gd proportions in the sample. Note the moments of nanoparticles initially rise at lower range of temperatures and saturation magnetization shows minimal variation at Curie temperature on right side of the curves (ASME IMEC Seattle meeting 2008: Presenter: Dr CJ Chen IMECE2007-43617).

Table 2. The Table shows specific T1 relaxivities of these different nanometal sample concentrations at different temperatures in the range of $41^{\circ} \mathrm{C}$ specifically near to the Curie temperature.

\begin{tabular}{|l|ccc|}
\hline $\begin{array}{l}\text { Concentration } \\
(\text { in } \mu \mathrm{g} / \mathrm{ml})\end{array}$ & $\begin{array}{c}\text { Temperature } \\
\left({ }^{\circ} \mathrm{C}\right)\end{array}$ & $\begin{array}{c}1 / \mathrm{T} 2 \\
(\mathrm{~ms})\end{array}$ & $\begin{array}{c}1 / \mathrm{T} 1 \\
(\mathrm{~ms})\end{array}$ \\
\hline Iron: & & & \\
200 & 41 & 0.264 & 0.168 \\
400 & & 0.283 & 0.154 \\
1000 & & 0.324 & 0.148 \\
Gadolinium: & & & \\
200 & 41 & 0.298 & 0.151 \\
400 & & 0.362 & 0.136 \\
1000 & & 0.284 & 0.153 \\
Zinc: & & & \\
200 & & 0.375 & 0.134 \\
400 & 41 & 0.286 & 0.152 \\
1000 & & 0.304 & 0.150 \\
Lanthanum: & & & \\
200 & 41 & 0.274 & 0.156 \\
400 & & 0.293 & 0.152 \\
1000 & & 0.376 & 0.133 \\
Gold: & & & \\
200 & & 0.276 & 0.138 \\
400 & & 0.285 & 0.151 \\
1000 & 41 & 0.385 & \\
& & & \\
\hline
\end{tabular}

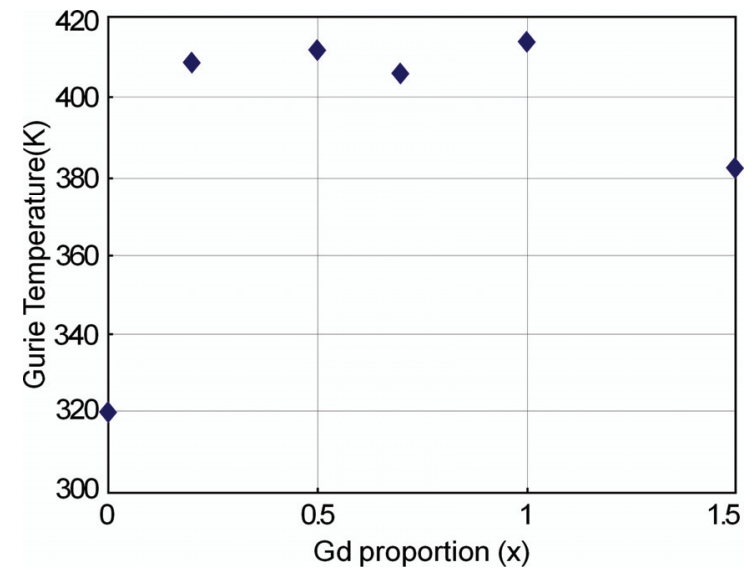

Figure 9. The figure shows variation of Curie temperature at different gadolinium proportion in the magnetic particle samples (ASME IMEC Seattle meeting 2008: Presenter: Dr CJ Chen IMECE2007-43617).

\subsection{Temperature Dependence of Magnetization}

The temperature dependence of magnetization was observed by using a Quantum Design SQUID. For this a constant field of $100 \mathrm{G}$ was applied and the moment was measured by varying the temperature from $0 \mathrm{~K}$ to $450 \mathrm{~K}$. Figure 8 shows the superimposed temperature dependence on Magnetization plots for all the samples. The $\mathrm{Cu}$ rie temperature was calculated by extrapolation of the linear sections of the temperature dependence plots [18]. The variation in Curie temperature with increasing Gd proportion is plotted in Figure 9.

\subsection{Proton NMR peaks and Relaxation Con- stants of Nanoparticles}

Magnetic particles Manganese (Mn), Zinc (Zn), Gadolinium $(\mathrm{Gd}), \operatorname{Gold}(\mathrm{Au})$ and $\operatorname{Iron}(\mathrm{Fe})$ with proportion $(\mathrm{x}=0.5)$ in the samples at different concentrations of 200,400 , $1000 \mu \mathrm{g} / \mathrm{ml}$ were tested at different temperatures of $41^{\circ} \mathrm{C}$. The NMR peak were used to calculated $1 / \mathrm{T} 1$ and $1 / \mathrm{T} 2$ as shown in Table 2 . These peak patterns also indicated the reasonable limit of temperature between $40-42^{\circ} \mathrm{C}$ as optimal temperature range for $\mathrm{T} 1$ measurement. The concentration of magnetic particles in the range of 200-400 $\mu \mathrm{g} / \mathrm{ml}$ and below $1000 \mu \mathrm{g} / \mathrm{ml}$ was sufficient to measure T1 relaxation constants of particles. 
Table 3. Emerging thermal mapping hybrid applications of thermal mapping and hyperthermia monitoring. Different techniques of temperature mapping with hyperthermia monitoring are shown with asterisk for their routine use *; research use **; and in infancy status ${ }^{* * *}$ in use.

\begin{tabular}{lc}
\hline $\begin{array}{l}\text { Hybrid Modality of thermal } \\
\text { Mapping with hyperthermia } \\
\text { Unit attached }\end{array}$ & $\begin{array}{l}\text { Physical property } \\
\text { for thermal mapping }\end{array}$ \\
\hline Ultrasound-MR* & \\
X-ray and MRI* & Echo and moment \\
Optical and MRI** & Attenuation/moment \\
PET and CT** & Ligand and moment \\
PET and MRI** & SUV and attenuation \\
Optical/Molecular imaging*** & SUV and moment \\
& Ligand specificity
\end{tabular}

\section{DISCUSSION}

In this study, Gd, Mn, Zn, ferrites with various Gd proportions were analyzed to study the effect on the magnetic properties of these particles and also to find a combination which will result in particles having a Curie temperature suitable for hyperthermia application. TEM suggested the size of these particles in the range of 20 $\mathrm{nm}$ and polymer PEG encapsulated nanospheres were measured in the range of 50-70 $\mathrm{nm}$. The particles were distinct by XRD peak patterns of zinc, Gd and iron oxide suggesting their distinct identity after PEG encapsulation in the nanospheres. In the following section different debated views are displayed for tumor temperature rise by magnetic nanoparticles $\mathrm{Mn}, \mathrm{Zn}, \mathrm{Gd}, \mathrm{Au}, \mathrm{La}, \mathrm{Fe}$ usable by multimodal imaging techniques.

A simplistic approach of tumor cell metabolic integrity loss associated with ATP loss, energy depletion, hypoxia leading to inflammation by lysosomal enzyme stimulation and cytokine synergy was presumed as proinflammatory cytokines and nitric oxide (NO) responsible for tumor cell killing. Under the condition of hyperthermia, the expression of pro-inflammatory cytokine (e.g. IL-6) and inhibitory (anti-inflammatory) cytokine(IL-10) were regulated. These temperaturedependent changes in the expression of IL-10 may imply an important clinical marker for hyperthermia-related tumor cell killing [28]. However, the mechanistic relation among temperature rise in tumor cells, energy depletion, and inflammation is still unclear. Heat shock treatment, inducing heat shock protein synthesis, also affected the regulation of cytosolic $\mathrm{I}-\kappa \mathrm{B}$ and translocation of NF- $\kappa \mathrm{B}$ into the nucleus [29].

The hysteresis curves showed sigmoid behavior of these nanoparticles and its magnitude dependent on temperature rise till temperature of particles reaches Curie temperature during AC vibrating resonator application. It suggests the hysteresis as major contributor at peculiar temperature end point so called "Curie temperature" in heating up to $70 \%$ of total heat as described in said section "Heat calculation of hyperthermia". The similar behavior was reported earlier without much information
Table 4. Potential newer nanoparticles used in thermal mapping and hyperthermia monitoring. Different nanoparticle composites are shown with their use in thermal mapping technique and possible use in hyperthermia monitoring. The potential nanoparticles in hyperthermia use are shown with + plus sign for routine use +++ , research use ++ , infancy state + or not established - at present.

\begin{tabular}{llc}
\hline Nanoparticles & Thermal mapping & Hyperthermia \\
\hline Zinc-Gadolinium-Ferrite & MRI, CT & + \\
Gold-Ferrite & MRI, CT & ++ \\
Gadolinium-Ferrite & MRI, CT & +++ \\
Lanthanum-Ferrite & MRI & + \\
Calcium & Optical, Molecular & + \\
Gd-Mn-Zn-Ferrite & MRI, CT & +++ \\
Mn-Gd-Ce & MRI & -- \\
La-Sr-Mn & MRI & -- \\
Nanoparticulate agents & MRI & -- \\
& & \\
\hline
\end{tabular}

of contribution in heating effect of tumors [15].

It was observed that the saturation magnetization of the particles drop with increasing Gd proportion. The initial increase in the saturation magnetization can be explained by considering that the $\mathrm{Gd}^{3+}$ ions have a large spin magnetic moment per atom $(7 \mu \mathrm{B})$ as compared to that of $\mathrm{Fe}^{3+}$ ion $(5 \mu \mathrm{B})$ [20-23]. Addition of $\mathrm{Gd}^{3+}$ ions results in their occupancy of the octahedral sites. The preference for octahedral sites maybe attributed to their large ionic radii. Since the ionic radii of the $\mathrm{Gd}^{3+}$ ions are large, there is a decrease in the distance between these and the oxygen ions when adding Gd ions [24]. As a result the ions at the octahedral sites no longer have their moments parallel to each other.

A part of these ions have moments aligned antiparallel to the other atoms on these octahedral sites. This results in a reduction in the net magnetic moment of the octahedral atoms. As the Gd substitution is increased, more and more octahedral atoms have their moments as antiparallel. As a result the saturation magnetization drops. Upadhyay et al. [23] have synthesized Gd substituted $\mathrm{Mn}-\mathrm{Zn}$ Ferrite nanoparticles using chemical coprecipitation. They observed an increase in the pyromagnetic co-efficient $(H T M) /(\partial \partial)$ of the resultant particles. The increase in the pyromagnetic co-efficient is desirable because it results in a steeper slope of the magnetization $\mathrm{v} / \mathrm{s}$ temperature plot which in turn ensures that the magnetization decreases rapidly as the temperature approaches the Curie temperature. This rapid decrease in magnetization means that the particles are heated up faster at temperatures below the Curie temperature and suddenly stop being heated near the Curie temperature which is a desirable property for Hyperthermia application. From Fig. 5 it can be seen that there is an increase in Curie temperature with Gd substitution.

Magnetic nanoparticles have found utility in many biological applications, including imaging, cancer therapy, drug delivery, sensing and hyperthermia for tumor therapy. In general, hyperthermia raises the tissue temperature between 41.5 - 46 degrees Celsius to kill can- 
cerous cells while preserving the normal cells. Several nanoparticles such as gold, zinc, gadolinium, lanthanum, and calcium have emerged as potential hyperthermia agents. Recently, new composite materials such as Mn$\mathrm{Zn}-\mathrm{Fe}, \mathrm{Co}-\mathrm{Gd}-\mathrm{Zn}$ and $\mathrm{Zn}-\mathrm{Gd}-\mathrm{Fe}$ nanoparticles with stable magnetic behavior have replaced magnetic oxides for use in hyperthermia at our lab. These composites generated sufficient heat at room temperature and stop heating at the Curie temperature $T_{c}$ of the respective nanoparticle system.

Gold nanoparticles (AuNP) killing the cancer cells was first reported by Hainfeld [98]. However, after injecting gold particles in animals and irradiation them by $250 \mathrm{kV}, \mathrm{X}$-rays caused tumor shrinkage and enhanced survival rate by four fold. The major challenge was localization of gold particles because of vascular leakage in the tumor but maximized particles entry in the tumor. Still, there are ample potential evidences in favor of gold enhanced x-ray hyperthermia in tumor treatment by killing. The application of gold particles in nanomedicine is its promise in radiotherapy of cancer. The Au-198 $\left(\beta_{\max }\right.$ $=0.96 \mathrm{MeV} ; \mathrm{t} / 2=2.7$ days $)$ and Au-199 $\left(\beta_{\max }=0.46\right.$ $\mathrm{MeV} ; \mathrm{t} / 2=3.14$ days) make them suitable in radiotherapy. In addition, gold particles display gamma emissions for dosimetry and pharmacokinetic studies. Therapeutic agents derived from gold particles provide a higher radioactivity dose to tumor sites. Furthermore, tumorspecific nanotherapeutic agents as a nanoparticle while tagged with peptides selective to receptors and overexpressed by tumor concentration offer another advantage. The gold nanoradioisotopes encapsulated within a nanocomposite device were reported as vehicles to transport radioactive particles to tumor sites. In this approach, particle size and number play a significant role such as nanocomposites made of monodisperse hybrid radioactive gold nanoparticles immobilized by dendritic polyamidoamine matrix prepared by reaction of polymer and tetrachloroaurate $\mathrm{HAuCl}_{4}$ solution. The salt formation between these solutions ensured the effective encapsulation of gold within the matrix using neutron irradiation in mice B 16 melanoma, prostate DU 145, human KB squamous cell carcinoma xenograft models. The property of polymer with $\beta$ emitting Au-198 enriched nano-device proved useful in tumor therapy. Moreover, the polymer enhances the stealthiness of magnetic nanoparticles by preventing macrophage recognition of particles as less toxic and resisting oxidation to make them valuable in multifunctional hyperthermia and imaging modalities. Another issue in tumor treatment is delivery of chemo-, gene-, radiotherapeutic agents within gold nanoparticles. It becomes effective as a tumor killing and targeted delivery tool.

These multicoponent particles are made of $\mathrm{Zn}_{\mathrm{x}} \mathrm{Mn}_{(1-}$ ${ }_{x)} \mathrm{Fe}_{3} \mathrm{O}_{4}$ and $\mathrm{Mn}_{\mathrm{x}} \mathrm{Zn}_{\mathrm{x}} \mathrm{Gd}_{\mathrm{x}} \mathrm{Fe}_{(2-\mathrm{x})} \mathrm{O}_{4}$ composites synthesized by physical and chemical co-precipitation methods. These particles displayed the increased tissue temperature and hyperthermia nature. Additionally particles in the form of $\mathrm{Ni}_{(1-\mathrm{x})} \mathrm{Cr}_{\mathrm{x}}$ were also formed. These particles may be encapsulated in thermo-sensitive polymer that dissolves when melted. The magnetic $\mathrm{Mn}-\mathrm{Zn}$ ferrite and Gd substituted Mn-Zn ferrite particles synthesized by the chemical co-precipitation method exhibited a specific behavior applicable in hyperthermia.

Paramagnetic gadolinium offers the excellent detection limit using contrast injection of $15 \mathrm{gm} / \mathrm{gm}$ tissue in clinical imaging. In our lab, these particles were encapsulated in thermosensitive polymer that dissolves after melting. These nanosized particles exhibited specific behavior of magnetic moments at Curie temperature and provide a window to evaluate their heating effect in tissue. Addition of $\mathrm{Gd}^{3+}$ ions up to proportions of $\mathrm{x}=0.5$ results in an increase in the net moment. Further addition of the $\mathrm{Gd}^{3+}$ ions results in a decrease in the net moment or saturation magnetization (SM). To prove it, the nanoparticle complex Mn-Gd-Ce was characterized by placing particles in a tube and its temperature increase with time as shown in Figure 9. The SM increases as temperature rises close to Curie temperature initially and subsequently SM decreases at temperature higher than the Curie temperature at increasing proportion of Gadolinium. Recently, nanosize Gd substituted Mn-Zn ferrite particles have been synthesized by a chemical coprecipitation method. These particles were mostly soft-magnetic. Gd substituted Mn-Zn Ferrite nanoparticles using chemical co-precipitation demonstrate an increase in the pyromagnetic co-efficient $(\mathrm{HTM}) /(\partial \partial)$ of the resultant particles. The increase in the pyromagnetic coefficient is desirable because it results in a steeper slope of the magnetization $\mathrm{v} / \mathrm{s}$ temperature plot which in turn ensures that the magnetization decreases rapidly as the temperature approaches the Curie temperature.

The Silica-Coated Lanthanum-Strontium Manganite Particles were prepared suited for hyperthermia. The core-comprising LaSr-manganites with different stoichiometries, ranging from $\mathrm{La}_{0.5} \mathrm{Sr}_{0.5} \mathrm{MnO}_{3+\delta}$ to $\mathrm{LaMnO}_{3+\delta}$, were synthesized as silica-coated magnetic particles with designable Curie temperature, offering a wide range of possibilities of adapting the material to practical instrumental setups in drug delivery and hyperthermia treatments. The relationship was based on temperature dependence of the proton chemical shift of water $\leq 0.01 \mathrm{ppm} /{ }^{\circ} \mathrm{C}$ at $\leq 0.7 \mathrm{~Hz}$ at $1.5 \mathrm{~T}$ for $1{ }^{\circ} \mathrm{C}$ temperature change using lanthanide complexes.

Malignant Hyperthermia ( $\mathrm{MH})$ is a hypermetabolic syndrome that results from the altered control of sarcoplasmic reticulum (SR) $\mathrm{Ca}^{2+}$ release. Recent study established the imaging of cytosolic $\left[\mathrm{Ca}^{2+}\right]\left(\left[\mathrm{Ca}^{2+}\right]_{\mathrm{c}}\right)$ in single cells grown from human skeletal muscle biopsies and in $\mathrm{H} 9 \mathrm{c} 2$ myotubes in response to a low dose of halothane on the cells derived from $\mathrm{MH}$ susceptible patients. The $\mathrm{Ca}^{2+}$ imaging in single cells is a promising candidate for the development of a new diagnostic and hyperthermia procedure of $\mathrm{MH}$.

Recent improvements in optical imaging techniques now allow these microdomains to be visualized as single channel calcium fluorescence transients (SCCaFTs), 
providing information about channel properties to monitor the activity and localization of microdomain calcium complexes. Other emerging possibilities are MR molecular imaging by gadolinium based perfluorocarbons, integrins, fibrins, and monoclonal antibody labeled nanoparticles. Recently remarkable progress is reported in real-time monitoring of thermal therapy and ablation using multimodal imaging techniques.

The emergence of a newer class of nanoparticles as contrast agents have created the desire of localized tumor molecular imaging based monitoring of tumor hyperthermia and molecular mapping responsible for thermal heating of a tumor as represented in Table 4. However, multimodal and multifunctional approaches of hyperthermia monitoring and thermal mapping are still in their infancy to use them in routine. The present growing interest of thermal mapping and hyperthermia monitoring is to achieve a rapid thermotherapy heating effect over focused tumor areas accurately by molecular imaging techniques.

Regional hyperthermia in combination with chemotherapy or/and radiotherapy has become state of the art as effective hyperthermia for locally advanced deepseated tumors as shown in Table 3. The thermometry using 3-dimensional US-MR-hyperthermia hybridsystem was installed and tested in phantoms and under clinical conditions in patients. The simultaneous MRimaging and T1-relaxometry at 0.2 Tesla during RF heating was performed using $\mathrm{T} 1$ sensitive pulse sequences to serve a basis for non-invasive MR-thermometry. The subtraction of $\mathrm{T} 1$ parameter maps before and during heating visualized the changes in $\mathrm{T} 1$. The patterns of $\mathrm{T} 1$ relaxation changes during hyperthermia treatment may prove to be useful for spatially resolved thermometry and thus help improve the hyperthermia therapy.

\section{CONCLUSION}

Nanosize Mn-Zn ferrite and Gd substituted Mn-Zn ferrite particles have been synthesized by chemical coprecipitation method. The samples are observed to be soft-magnetic. Addition of $\mathrm{Gd}^{3+}$ ions up to proportions of $\mathrm{x}=0.5$ results in an increase in the net moment. Further addition of the $\mathrm{Gd}^{3+}$ ions results in a decrease in the net moment. The saturation magnetization increases then decreases with increasing proportion of Gadolinium. The Curie temperature increases with addition of Gadolinium. But addition of $\mathrm{Gd}$ in proportions more than $\mathrm{x}=1.0$ results in a decrease in Curie temperature. $\mathrm{Mn}_{0.5} \cdot \mathrm{Zn}_{0.5} \cdot$ Ferrite ferromagnetic nanomagneticparticles showed sharp ferromagnetic to paramagnetic transition behavior at specific temperature.

\section{ACKNOWLEDGEMENTS}

The project was partially funded by a grant from the Florida State University Research Foundation to the Center of Nanomagnetics and Biotechnology. Tallahassee, FL. Authors are grateful for assistance of Virendra Mohite and Dr Saleh Hayek for their graduate work in this grant. The data was partly presented by Dr Chen CJ at ASME IMEC Seattle meeting 2008 IMECE2007-43617NSTI meeting 2007.

\section{REFERENCES}

[1] W. Busch, (1966), Rheinisch-Westfael. Akad. Nat. Ing. Wirtschaftswiss Vortr. 23, 28.

[2] W. B. Coley, (1993) Am. J. Med. Sci. 105488.

[3] G. Crile, (1963) Cancer Res. 23, 372.

[4] R. Cavaliere, E. C. Ciocatta, B. C Giovanella, C. Heidelberger, (1967) R. D. et al. Cancer 20, 1351.

[5] J. W. Hand and G. ter Haar, (1981) Br. J. Radiol. 54, 443.

[6] D. A. Christensen and C. H. Durney, J. Microwave Power 16 (1981) 89).

[7] J. W. Strohbehn and E. B. Douple, (1984) IEEE Trans. Biomed. Eng. BME-31, 779.

[8] C. C. Vernon, J. W. Hand, S. B. Field, et al. (1996) Int. J. Rad. Oncol. Biol. Phys. 35, 731-744.

[9] J. Van der Zee, (2002) Annals Oncology 13, 1173-1184.

[10] G. Multhoff, C. Botzler, M. Wiesnet, E. Muller, T. Meier, (1995) W. Wilmanns and R. D. Issle, Int. J.Cancer 61, 272-279.

[11] B. Park, B. S. Koo, Y. K. Kim, M. K. Kim, (2002) Korean J Radiol, 3, 98-104.

[12] S. Hayek. Chapter 5. In:Ph.D thesis submitted to Florida State University, Tallahassee.

[13] R. Sharma. Chapter 4. In: Ph.D thesis submitted to Indian Institute of Technology, New Delhi.

[14] S. Deger, D. Boehmer, I. Turk, J. Roigas, V. Budach, S. Loening, (2002) European Urology, 42, 147-153.

[15] A. Jordan, R. Scholz, P. Wust, H. Fahling. R. Felix, (1999) J Mag Mag Matr, 201, 413-419.

[16] N. Brusentsov, L. Nikitin, T. Brusentsova, A. Kuznetsov, F. Bayburtskiy, L.Schumakov, N.Jurchenko, (2002) J. Mag. Mag. Matr., 252, 378-380.

[17] E. Auzans, D. Zins, M. M. Maiorov, E. Blums, R. Massart, (1999) Magn. Gidrodinamika 38, 78-86.

[18] E. Auzans, D. Zins, E. Blums, R. Massart, (1999) J. Mater. Sci. 34, $1253-1260$.

[19] E. Auzans, (1999) Mn-Zn ferrite nanoparticles for water- and hydrocarbone-based ferrofluids:preparation and properties, Thesis.

[20] C. Kittel, Intro. To Solid State Physics, John Wiley \& Sons, NY, 4 th Ed.

[21] G. Burns, (1985) Solid State Physics, Academic Press Inc.

[22] K. Schroder, (1978) Elec., Mag. And Thermal Properties of Solid Mat., Marcel Dekker Inc. NY.

[23] R. Kubo, T. Nagamiya, (1969) Solid State Physics, McGraw Hill.

[24] J. Smit And H. P. J. Wijn, Ferrites, John Wiley \& Sons, NY, 1959, p 139-142.

[25] R. V. Upadhyay, R.V. Mehta, K. Parekh, D. Shrinivas, R.P. Pant, (1999) J. Magn. Magn. Mater. 201,129 - 132.

[26] F. Settecase, M.S. Sussman, (2007) TPL Roberts, Contrast Media Mol Imaging, 2,50-54.

[27] R Hergt,S Dutz,R Muller,M Zeisberger.(2006) J Phys Cond Mat.18:S2919-S2934.

[28] T. Matsui, T. Kakeda, (2008.)J Neurotrauma.25(6):709-15.

[29] H. W. Chen, H. T. Kuo, S. J. Wang, T.S. Lu, R.C. Yang, (2005) Shock. 24(3):232-8. 